\title{
EVALUATION OF TERRESTRIAL LASER SCANNING FOR RICE GROWTH MONITORING
}

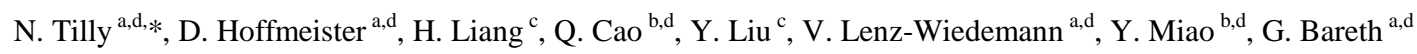 \\ ${ }^{\text {a }}$ Institute of Geography (GIS \& Remote Sensing Group), University of Cologne, 50923 Cologne, Germany - \\ (nora.tilly, dirk.hoffmeister, victoria.lenz, g.bareth)@uni-koeln.de \\ ${ }^{\mathrm{b}}$ Department of Plant Nutrition, China Agricultural University, 100193 Beijing, China, - \\ (qiangcao,ymiao)@cau.edu.cn \\ ${ }^{\mathrm{c}}$ Five Star Electronic Technologies Co. Ltd., Fengtai District, Fangchengyuanyiqu, Building17, Riyuetiandi B,100078 \\ Beijing - liuyj0001@yahoo.com, china.lh@gmail.com \\ ${ }^{\mathrm{d}}$ International Center for Agro-Informatics and Sustainable Development (ICASD), www.icasd.org
}

KEY WORDS: TLS, Multitemporal, Agriculture, Crop, Change Detection, Monitoring

\begin{abstract}
:
The rapidly growing world population and the resulting pressure on the efficiency of agriculture require a sustainable development of intensive field management with regard to natural resources. In this context, the use of non-destructive remote sensing technologies to monitor status and change detection of plant growth is in the focus of research and application. In this contribution, we evaluate the applicability of multitemporal terrestrial laser scanning (TLS) for rice growth monitoring. The test sites are located around Jiansanjiang in Heilongjiang Province in the far northeast of China. The focus of the field experiment was on different nitrogen fertilizer inputs during the growing period in 2011. To realize the monitoring approach, three campaigns were carried out during the vegetative stage of rice plants. For all campaigns the terrestrial laser scanner Riegl VZ-1000 was used. The achieved knowledge can be described in two parts. First, for each date the variability of plant height and biomass is detectable for the whole experiment field and - more important - between the plots. Furthermore, differences in height and biomass related to edge effects can be investigated for every single plot. The spatial distribution is visualized by Crop Surface Models (CSM), which are digital surface models with a high resolution and accuracy achieved by the interpolation of the 3D point clouds. Secondly, the multitemporal surveying approach enables the monitoring of the growth rate of the rice plants. Additionally, it is possible to detect and analyze as well the spatial distribution of the changes by comparing the CSMs. Our results show that TLS is a suitable and promising method for rice growth monitoring. Furthermore, the contemporaneous surveying with other sensors enables us to validate our measurements and bares opportunities for further enhancements.
\end{abstract}

\section{INTRODUCTION}

The principle of Light Detection and Ranging (LIDAR) systems is the computation of distances between a sensor and a target with a laser beam (Jensen, 2007). This is possible by (i) measuring the time between transmitting and receiving a pulsed signal, (ii) calculating the phase shift in a sinusoidal continuous beam, or (iii) detecting a laser dot on a target with a camera and calculate the distance from the triangle between transmitter, camera, and target (Shan \& Toth, 2009; Kraus, 2004). Thus, highly accurate $3 \mathrm{D}$ point clouds are obtained from the achieved information. Depending on the used platform, it can be distinguished between Airborne Laser Scanning (ALS), Terrestrial Laser Scanning (TLS), and Mobile Laser Scanning (MLS) (Vosselmann \& Maas, 2010). The methods differ in their accuracy, spatial resolution, covered area, and measuring rate.

Generally, the investigation and monitoring of the earth's surface by remote sensing techniques is object of research in various fields of interests. However, in the field of agriculture, laser scanning is only used for a few applications. McKinion et al. (2010) established yield stability maps for cotton and corn fields over a period of three years with ALS measurements. At a smaller scale, Eitel et al. (2011) analysed the nitrogen status of spring wheat using a TLS system with a green scanning laser. Based on a previous study (Eitel et al., 2010), they detected the ability to quantify the crop nitrogen status by the relationship between leaf chlorophyll and reflected green laser light. In the context of precision farming, Saeys et al. (2009) mounted a laser scanner on a combine harvester to estimate the crop density while driving. The driving speed can be adjusted automatically in order to maximize the capacity of the harvester. The usability of multi-temporal TLS to detect 3D crop changes, is presented by Hoffmeister et al. (2010). In this study, patterns in the distribution of height differences within a sugar-beet field were detected with a timeof-flight laser scanner and visualized in Crop Surface Models (CSM) and Crop Volume Models (CVM). Ehlert et al. (2008, 2009) established a measuring system with a triangulation and a time-of-flight scanner. The estimated mean crop heights (oilseed rape, winter rye, winter wheat, and grassland) were correlated to fresh and dry biomass with good results $\left(R^{2}=0.75\right.$ to 0.99). The impact of different nitrogen fertilizer rates on various cereals (barley, oat, and wheat) was investigated with a phase-shift scanner by Lumme et al. (2008). They found a good correlation between plant height and grain yield $\left(\mathrm{R}^{2}=0.88\right.$ to $0.99)$.

The structure of rice plants is similar to the investigated cereals, which suggests that laser scanning can also be used to determine rice crop properties. Moreover, the importance of

\footnotetext{
* Corresponding author: nora.tilly@uni-koeln.de
} 
rice cultivation increases because it is a staple food, in particular for the rapidly growing Asian population. Casanova et al. (1998) already presented a study about monitoring rice during the growing season with a hand-held radiometer. Their conclusion was that only biomass can be predicted with a high accuracy $\left(\mathrm{R}^{2}=0.97\right)$. The possibility to estimate the leaf area index (LAI) from spectral data is limited $\left(\mathrm{R}^{2}=0.67\right)$. Recent studies focus on deriving different vegetation properties, like the LAI and the green leaf chlorophyll density (GLCD), from remote sensing data. Therefore, Yang et al. (2011) compared different transformations for reflectance data, obtained from a spectroradiometer, concerning their capability of predicting rice biophysical parameters. In another study Ryu et al. (2011) used an airborne hyperspectral sensor to determine the nitrogen content of rice plants at the heading stage. In the context of rice growth monitoring, Koppe et al. (2012) demonstrated the potential of multi-temporal and dualpolarimetric TerraSAR-X data based on a survey carried out in the same region observed in our study. However, any studies about TLS measurements for rice exist at this stage.

Thus, we evaluate the applicability of multitemporal TLS for rice growth monitoring in this approach. In contrast to other presented studies using phase-shift or optical probe sensors, a time-of-flight sensor was used. The surveys were carried out on test sites related to the ICASD project. The International Center for Agro-Informatics and Sustainable Development (ICASD) was founded in 2009 as a cooperative research center between the China Agricultural University, Beijing and the University of Cologne, Germany (ICASD, 2012). Besides the development of an open, international, and multidisciplinary platform for agroinformatics and sustainable development, one of the major aims is the application of information sciences and technologies in the field of agriculture.

\section{METHODS}

\subsection{Study area}

We conducted our surveys on test fields close to the city Jiansanjiang (N 47 $13{ }^{\prime} 54^{\prime \prime}$, E $\left.132^{\circ} 38^{\prime} 53^{\prime \prime}\right)$ in Heilongjiang Province in the far northeast of China. The Province with a continental monsoon climate is an important basis for agricultural products (Gao \& Liu, 2011).

The focus of the monitored field experiment was on different nitrogen fertilizer inputs during the growing period. One half of the paddy rice field with a spatial extent of $60 \mathrm{~m}$ by $60 \mathrm{~m}$ was cultivated with the rice variety Kongyul31, the other one with Longjing21. Furthermore, nine different treatments were repeated thrice for both rice varieties. The treatments differ in the amount of applied nitrogen fertilizer during the early and middle growing period. Thus, the area was divided into 54 plots (10 $\mathrm{m}$ by $7 \mathrm{~m})$.

\subsection{Surveys}

For all field campaigns the terrestrial laser scanner Riegl VZ-1000 (Riegl LMS GmbH, 2011), provided by Five Star Electronic Technologies, located in Beijing, was used. The narrow infrared laser beam with online full waveform analysis and echo digitization enables the fast recording of high-accurate 3D point clouds. Additionally, a digital camera, Nikon D700, was mounted on the laser scanner. From the recorded RGBphotos the point clouds gained from the laser scanner can be colorized and the corresponding surfaces can be textured.

The instrument was fixed on a tripod, which raises the sensor up to $1.5 \mathrm{~m}$ above ground. Where possible, the whole setting was build up on a small trailer behind a tractor to achieve a greater height (cf. Figure 1). In order to capture the whole study area, nine scan positions were established. For the analyses presented in this paper, four of them were of major importance: Two positions without the trailer at the north edge and two positions with the trailer at the south edge of the investigated field.

For the monitoring approach, three campaigns were carried out on the $21^{\text {st }}$ of June, the $4^{\text {th }}$, and the $18^{\text {th }}$ of July 2011 . The chosen period is within the vegetative stage of the rice plants, when the stem elongation process takes place. Remarkable differences between the dates were assumed, due to the increase of tillers and plant height during this stage.

Moreover, common tie points in all scans are required to enable the merging of all scan positions in the postprocessing. Therefore, high reflective cylinders that can be easily detected by the laser scanner, were fixed on ranging poles build upon the dikes between the fields (Hoffmeister et al., 2010). The positions of these poles were marked with large nails in the first campaign. Thus, every scan of each date can be merged together by reestablishing the ranging poles for the other two campaigns. Furthermore, on each date the plant heights of eight to ten plants per plot were measured manually and plant samples were taken to evaluate the biomass of stem and leafs.

\subsection{Postprocessing}

For the postprocessing of the achieved data, Riegls software RiSCAN PRO, which is delivered with the laser scanner, was used. The first registration and merging of the scan positions was performed with an indirect registration method, based on the mentioned high reflective cylinders acting as tie points.

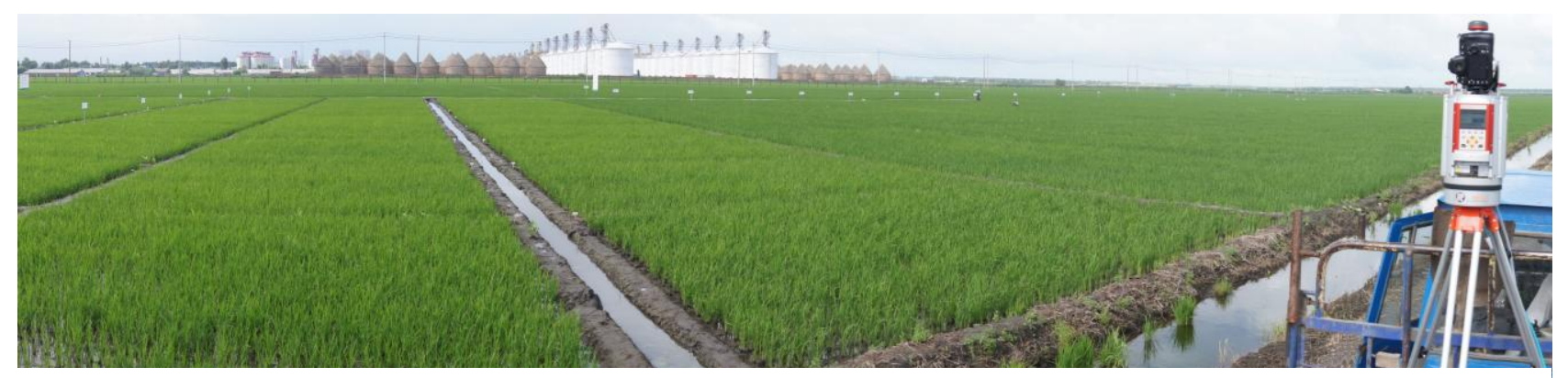

Figure 1. Overview of the investigated area from one scan position at the south edge of the field. On the right side the scanner with the tripod mounted on the small trailer can be seen. 
However, a further adjustment was applied, to enhance this registration result. Therefore, RiSCAN PRO offers the Multi Station Adjustment, which is based on the Iterative Closest Point (ICP) Algorithm (Besl \& McKay, 1992). The position and orientation of each scan position can be modified in multiple iterations.

Subsequently, the Area Of Interest (AOI) was manually extracted from the merged point cloud of each date. For an easier orientation and the distinction between fields and dikes, the point clouds were colorized from the recorded photos. Nevertheless, the point clouds still contain noise, caused by reflections on water or on small particles in the air. To reduce this, a further filtering based on reflectance, measured for each point during the data acquisition, was performed.

In a next step, the cleaned point clouds were interpolated in order to receive a digital terrain model (DTM), with a spatial resolution of $0.01 \mathrm{~m}$. For the calculation of the plant heights and the comparison of the results from the different dates a common reference surface is required. Usually a high accurate Digital Surface Model (DSM), achievable from scanning the AOI without any vegetation, is used. However, since such data were not obtained in this present study, we applied another method. In order to receive a reference surface similar to the real ground, the lowest parts in the point cloud from the first date, accordingly containing the least dense vegetation, were selected manually to interpolate a DSM. As it can be seen in Figure 2, the rice plants were small enough to clearly identify points on the ground. Finally, the Crop Surface Models (CSM) as the difference between DTM and DSM were established for each date. Likewise, the differences between the CSMs were calculated for growth monitoring.

Moreover, the height values, stored in the CSMs, were used to calculate the mean plant height and growth of each plot. In the following, growth is always defined as a difference in height. Additionally, the manually measured heights were averaged for each plot and correlated with the values from the CSMs to validate the results.

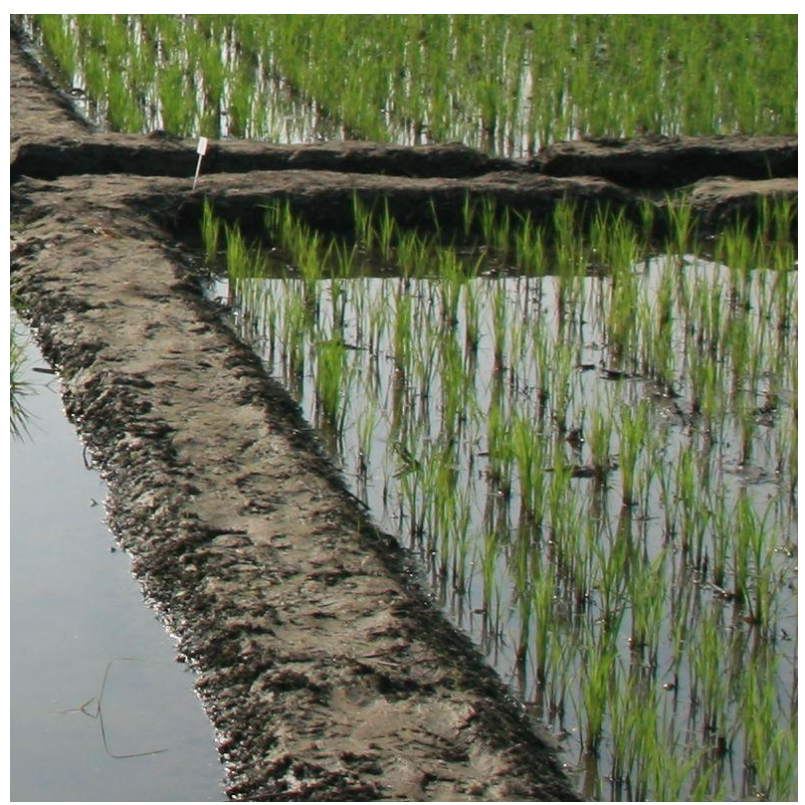

Figure 2. Photo of one corner of the investigated field, showing the least dense vegetation.

\section{RESULTS}

\subsection{Point Clouds}

As a first result, point clouds with high density and accuracy are derived. The merged and filtered datasets contain six to ten Mio. points for each date, just covering the AOI. This data was used to create the CSMs and calculate the mean plant height and growth for each plot.

\subsection{Crop Surface Models}

The received CSMs can be visualized as maps of height growth. Therefore, the height was calculated above the common reference surface, considered as DSM in this study. Figure 3 shows the maps for four selected plots of the same treatment. On the left side, two repetition plots of Kongyu131, on the right side two repetition plots of Longjing 21 are shown. For the presentation, the point clouds were interpolated, using the Inverse Distance Weighting (IDW) algorithm. The results are stored in raster data sets with a resolution of $0.01 \mathrm{~m}$.

The following statements can be made from the maps (Figure 3):

- The plant height of Longjing21 is - especially at the last date - higher than Kongyu131.

- In plot 163 the linear structure of the fields can be clearly seen.

- In plot 262 pronounced height differences within the plot can be detected.

The spatial distribution of the height difference between the CSMs, showing the plant growth, can also be visualized by the calculation of a new raster. As a result, varieties within the fields can be detected. Figure 4 shows the calculated height difference between two consecutive CSMs of two plots and hence the change in plant height between the first and second, respectively second and third date. For both plots an evenly distributed increase in height can be concluded. Moreover, a stronger increase of height can be detected for Longjing 21 (plot 232), especially for the period between the second and third date.

\subsection{Mean Plant Height and Biomass}

From the CSMs, the mean plant height for each single plot was calculated as the height above the common reference surface. Furthermore, the averaged manually measured plant heights were checked against the heights from the CSMs. For the statistical analyses 18 plots (nine of each rice variety), well distributed over the whole field, were chosen. Since the measurements from all dates were used, the analyses are based on 54 values. The height values comprise a great range (cf. Figure 5), which makes the regression more reliable. The correlation between the mean plant height achieved from the CSMs and the average measured plant height is very good $\left(\mathrm{R}^{2}=0.91\right)$. 


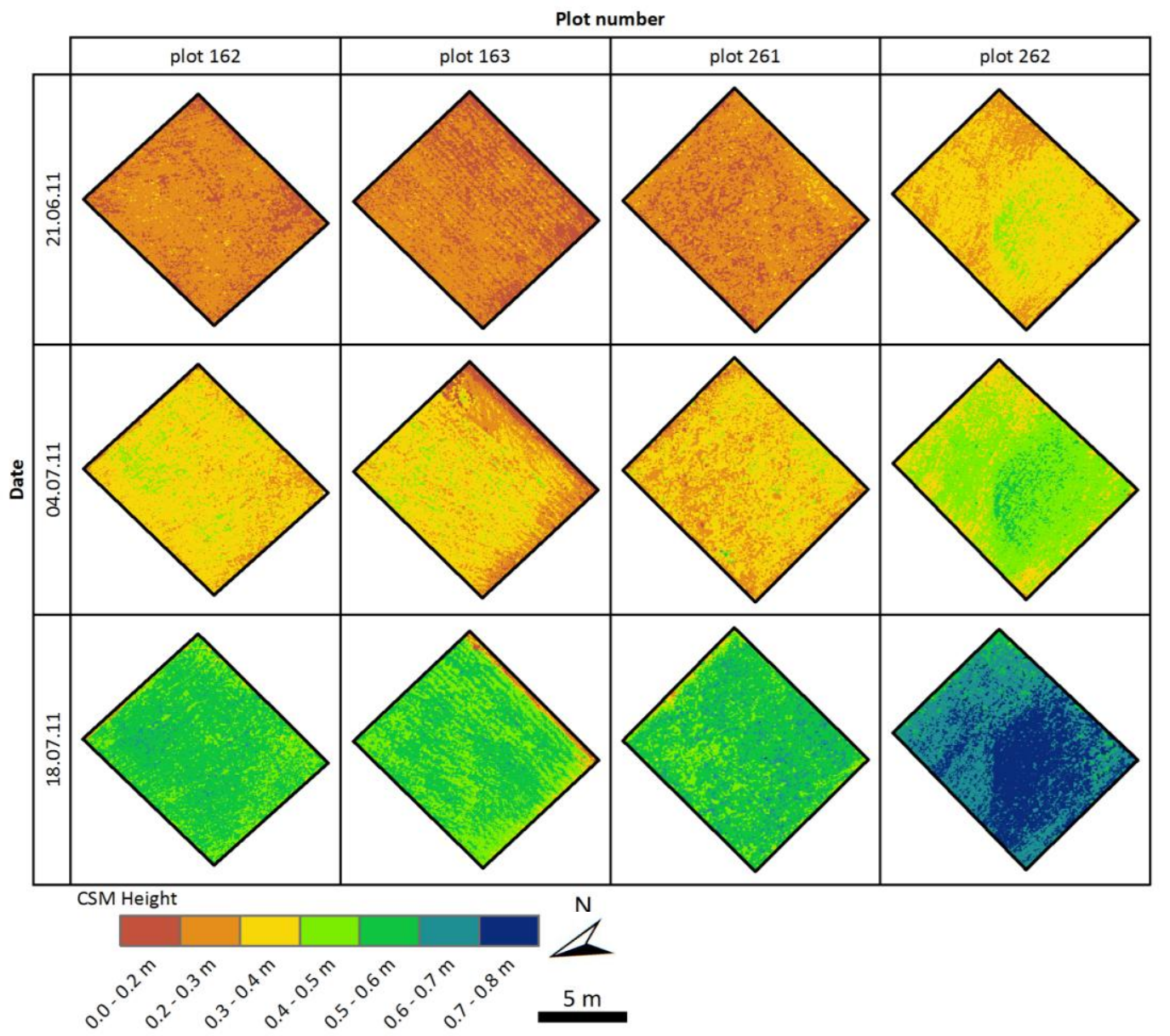

Figure 3. Maps of Crop Surface Models for four selected plots, showing the results for all three dates. The height is always calculated by subtracting the DSM of each date from the DTM.
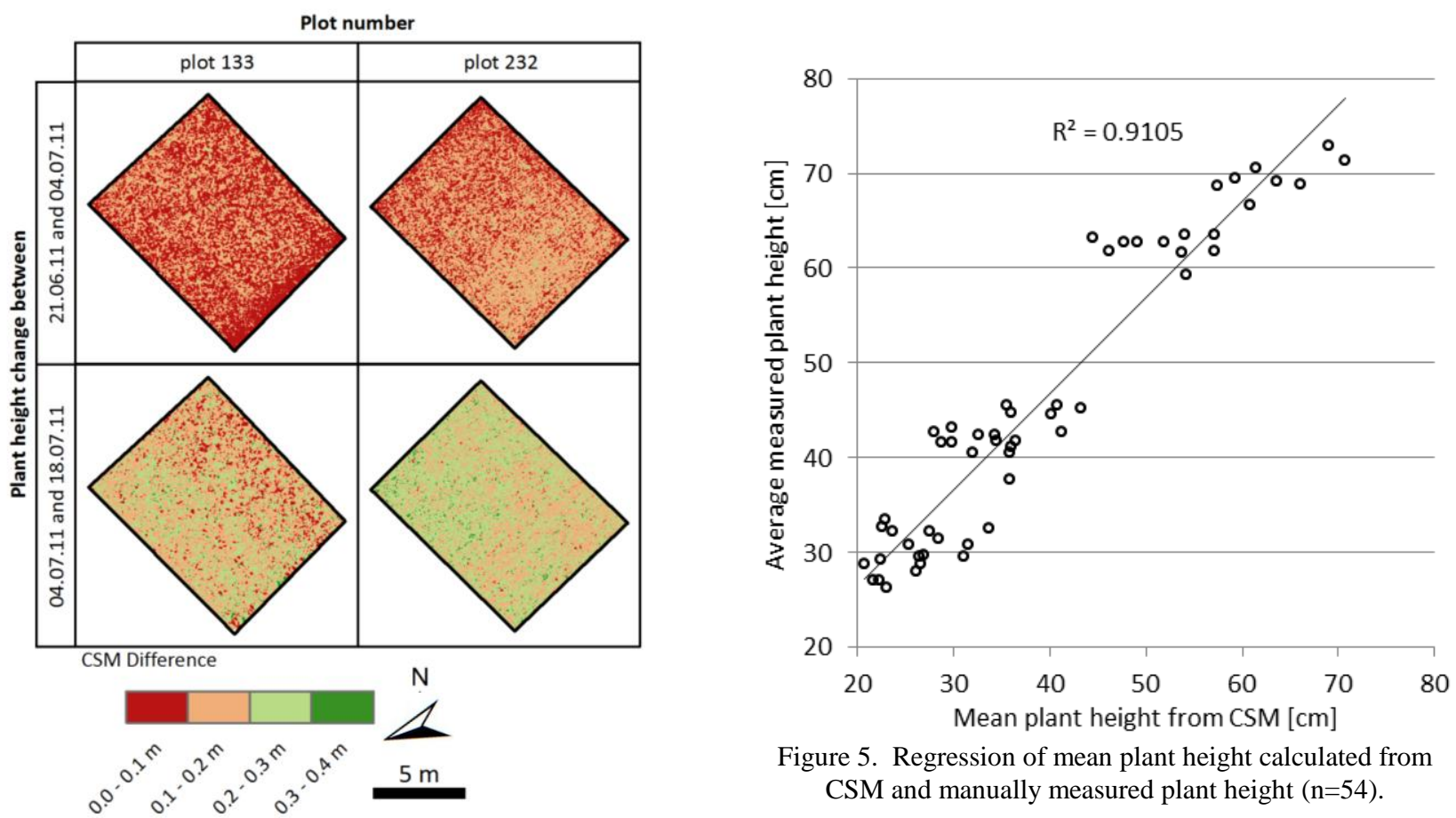

Figure 4. Maps of height difference between two consecutive CSMs.

Figure 5. Regression of mean plant height calculated from CSM and manually measured plant height $(n=54)$. 
Additionally, at each date plant samples from selected plots were taken and dry biomass (stem and leaf) was measured. Thus, the statistical analyses are based on 22 values. A very good correlation between plant height and dry biomass was achieved $\left(\mathrm{R}^{2}=0.88\right)$ and the regression line fits well (cf. Figure 6).

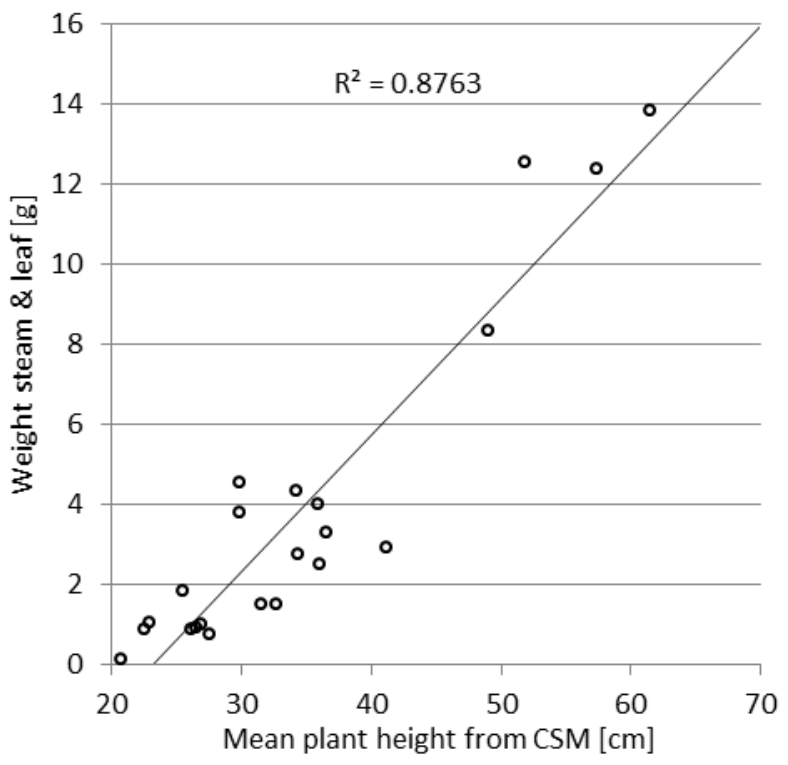

Figure 6. Regression of mean plant height calculated from CSM and biomass of stem and leaf $(n=22)$.

\subsection{Monitoring Approach}

The change in plant height was monitored by the calculation of differences between each CSM, similar to the calculation of the plant height above the reference surface. In order to verify the results, the average manually measured plant growth was calculated and compared to the outcomes from the CSMs. The coefficient of determination $\left(\mathrm{R}^{2}=0.86\right)$ is again very good. As it can be seen in Figure 7, the calculation of the regression line is based on values of a great range from 5 to $30 \mathrm{~cm}$, which reinforces the plausibility.

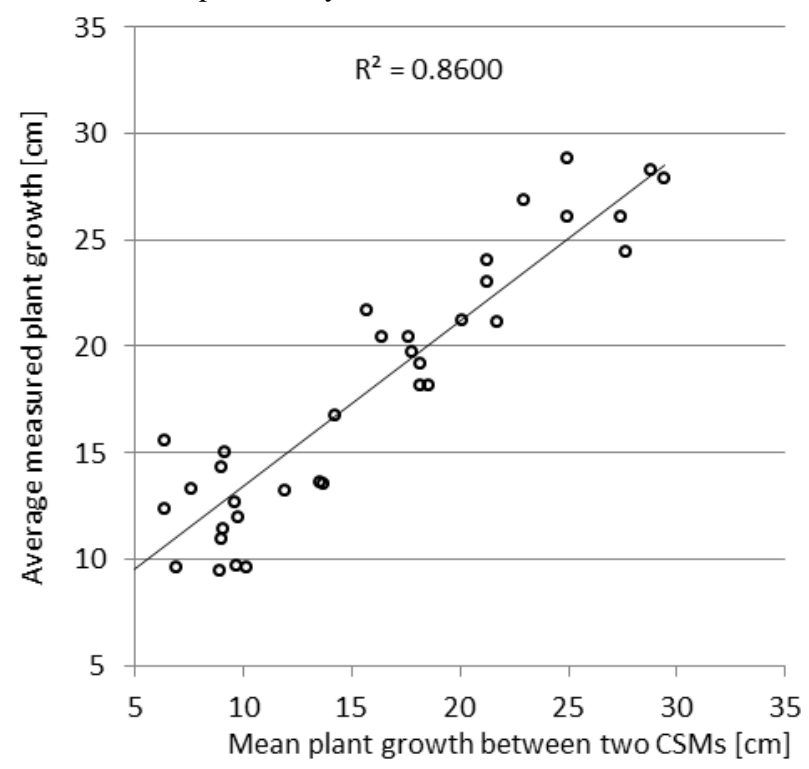

Figure 7. Regression of mean plant growth increase between two consecutive CSMs and average measured plant growth increase $(n=36)$.

\section{DISCUSSION}

First of all, the data acquisition with the laser scanner in the field worked very well. A major advantage of the TLS system is the easily achievable and fast data acquisition of the whole field, compared to Casanova et al. (1998), using a hand-held radiometer. Anyway, an accurate differentiation between each plot is possible with a higher spatial resolution than achievable with ALS (McKinion et al., 2010).

However, the compact and lightweight build-up of the Riegl VZ-1000 is therefore quite helpful. Ehlert et al. (2009) and Lumme et al. (2009) complain about problems with noise in the point clouds. This problem is always linked with TLS, due to wind and other effects. However, the time-of-flight scanner, used in this study, reduces this problem by the high measuring speed. Earlier studies with a comparable set-up (Hoffmeister et al., 2011), show already the usability of this method, but further improvement is still desirable. Nevertheless, some minor problems, like the transportation of the scanner on the dikes, have to be solved.

For the registration and merging of the scan positions, RiSCAN PRO offers appropriate tools. The indirect registration based on the tie points can be considerably improved by the ICP algorithm. By employing the MSA, the standard deviation error was decreased from $0.1 \mathrm{~m}$ to $0.05 \mathrm{~m}$. Furthermore, filtering based on the reflectance was helpful to remove noise from the point clouds. Thus, the time consuming manual postprocessing was remarkably accelerated. Furthermore, the scan positions where the setting could be build up on the trailer, profit from the greater height. This could be seen e.g. in the CSMs of plot 163 (cf. Figure 3). The plot was at the south edge of the investigated field, close to the scan positions with the trailer. Due to the higher perspective, the linear structure of the field is strongly visible.

Beside the well working method, the results show that our approach seems to be suitable for rice growth monitoring. The good correlation between the mean plant height calculated from the CSMs and the manually measured plant height $\left(\mathrm{R}^{2}=0.91\right)$ as well as the correlation with the dry biomass $\left(\mathrm{R}^{2}=0.88\right)$ show the accuracy of the achieved models. However, the accuracy of the data is comparable with those from the mentioned studies with cereals (Ehlert et al., 2008; 2009). Moreover, the correlation concerning the plant growth $\left(\mathrm{R}^{2}=0.86\right)$ confirms the suitability for the monitoring approach. The already mentioned study of Hoffmeister et al. (2011) with a comparable set-up, was carried out on sugar-beet fields. In contrast to rice, the more complex structure of the sugar-beet leaves impairs the correlation between mean height and dry biomass.

Moreover, the spatial distribution of variances within the CSM of one plot and between different CSMs can be detected. First, the CSMs from various repetitions can be compared. Secondly, for monitoring, CSMs from several time steps can be compared to receive information about plant growth. Again, compared to the studies on sugar-beet fields (Hoffmeister et al., 2010; 2011) spatial differences in height were detectable as well.

\section{CONCLUSION AND OUTLOOK}

The results presented in this contribution show the applicability for accurate capturing and monitoring of rice growth in terms of changes in plant height and biomass. These spatial patterns of 
plant growth are measureable by this method. A very good correlation between plant height and dry biomass enables the establishment of biomass calculations at a certain time step by this measurement technique. Furthermore, the visualization of the spatial distribution in a high resolution is possible.

For the future, two research approaches should be followed. First, TLS data could be linked to spectral data, acquired in the field (Li et al., 2010). Secondly, the usability of Unmanned Aerial Vehicles (UAV) for rice growth monitoring with laser scanning might be considered. As Bareth et al. (2011) mention, the Riegl LMS-Q160, developed for ALS, might be a promising device.

\section{ACKNOWLEDGEMENTS}

This work was financially supported by the International Bureau of the German Federal Ministry of Education and Research (BMBF, project number 01DO12013) and the German Research Foundation (DFG, project number BA 2062/8-1). We gratefully thank the Qixing Research and Development Centre, the Jiansanjiang Agricultural Research Station (both located in Heilongjiang Province, China) and Five Star Electronic Technologies (Beijing, China) for good cooperation. Furthermore, we like to thank RIEGL LMS GmbH (Horn, Austria) for continuous support.

\section{REFERENCES}

Bareth, G., Bolten, A. and Bendig, J., 2011. Potentials of lowcost mini-UAVs. In: Lenz-Wiedemann, V. and Bareth, G. (eds). Proc. of the Workshop on Remote Sensing Methods for Change Detection and Process Modelling, Cologne, Germany. Kölner Geographische Arbeiten, 192, pp. 1-8.

Besl, P.J. and McKay, N.D., 1992: A method for registration of 3-D shapes. IEEE Transactions on Pattern Analysis and Machine Intelligence, 14(2), pp. 239-256.

Casanova, D., Epema, G.F. and Goudriaan, J., 1998. Monitoring rice reflectance at field level for estimating biomass and LAI. Field Crops Reasearch, 55, pp. 83-92.

Ehlert, D., Adamek, R. and Horn, H.-J., 2009. Laser rangefinder-based measuring of crop biomass under field conditions. Precision Agriculture, 10(5), pp. 395-408. doi: 10.1007/s11119-009-9114-4.

Ehlert, D., Horn, H.-J. and Adamek, R., 2008. Measuring crop biomass density by laser triangulation. Computer and Eletronics in Agriculture (61), pp. 117-125.

Eitel, J.U.H., Vierling, L.A. and Long, D.S., 2010. Simultaneous measurements of plant structure and chlorophyll content in broadleaf saplings with a terrestrial laser scanner. Remote Sensing of Environment, 114, pp. 2229-2237.

Eitel, J.U.H., Vierling, L.A., Long, D.S. and Hunt, E.R., 2011. Early season remote sensing of wheat nitrogen status using a green scanning laser. Agriculture and Forest Meteorology, 151, pp. 1338-1345.

Gao, J. and Liu, Y., 2011. Climate warming and land use change in Heilongjiang Province, Northeast China. Applied Geography, 31, pp. 476-482.
Hoffmeister, D., Bolten, A., Curdt, C., Waldhoff, G. and Bareth, G., 2010. High resolution Crop Surface Models (CSM) and Crop Volume Models (CVM) on field level by terrestrial laserscanning. Proc. SPIE, 7840, 78400E. 6 p. doi: 10.1117/12.872315.

Hoffmeister, D., Curdt, C., Tilly, N., Bendig, J. and Bareth, G., 2011. 3D change detection of different sugar-beet types by multi-temporal terrestrial laser scanning. Proc. Int. Symposium on remote sensing and GIS methods for change detection and spatio-temporal modeling, Hong Kong, China.

ICASD, 2012. International Center for Agro-Informatics and Sustainable Development. Cologne, Germany. http://www.icasd.org (9. Jan 2012)

Jensen, J.R., 2007. Remote Sensing of the Environment. Upper Saddle River, USA.

Kraus, K., 2004. Photogrammetrie. Berlin, Germany.

Koppe, W., Gnyp, M., Hütt, C., Yao, Y., Miao, Y., Chen, X. and Bareth, G., 2012, in press. Rice monitoring with multitemporal and dual-polarimetric TerraSAR-X data. JARS.

Li, F., Miao, Y., Hennig, S.D., Gnyp, M.L., Chen, X., Jia, L. and Bareth, G., 2010. Evaluating hyperspectral vegetation indicies for estimating nitrogen concentration of winter wheat at different growth stages. Precision Agriculture, 11, pp. 335-357.

Lumme, J., Karjalainen, M., Kaartinen, H., Kukko, A., Hyyppä, J., Hyyppä, H., Jaakola, A. and Kleemola, J., 2008. Terrestrial Laser Scanning of agricultural crops. In: XXI. ISPRS Conference, 3.-11. July 2008. Beijing, China. pp. 563-566.

McKinion, J.M., Willers, J.L. and Jenkins, J.N., 2010. Spatial analyses to evaluate multi-crop yield stability for a field. Computers and Electronics in Agriculture, 70, pp. 187-198.

Riegl LMS GmbH, 2011. Datasheet Riegl VZ-1000. http://riegl.com/uploads/tx_pxpriegldownloads/10_DataSheet_ VZ1000_12-09-2011.pdf (9. Jan 2012)

Ryu, C., Suguri, M. and Umeda, M., 2011. Multivariate analysis of nitrogen content for rice at the heading stage using reflectance of airborne hyperspectral remote sensing. Field Crops Research, 122, pp. 214-224.

Saeys, W., Lenaerts, B., Craessaerts, G. and De Baerdemaeker, J., 2009. Estimation of the crop density of small grains using LiDAR sensors. Biosystems Engineering, 102, pp. 22-30.

Shan, J. and Toth, C.K., 2009. Topographic Laser Ranging and Scanning. Boca Raton, USA. pp. 1-28.

Vosselmann, G. and Maas, H.-G., 2010. Airborne and terrestrial laser scanning. Dunbeath, UK.

Yang, X.H., Huang, J.F., Wu, Y.P., Wang, J.W., Wang, P., Wang, X.M. and Huete, A.R., 2011. Estimating biophysical parameters of rice with remote sensing data using support vector machines. Science China Life Sciences, 54, pp. 272-281. 